\title{
APPLICATION OF POWER ULTRASOUND FOR NANO-PARTICLE SIZE REDUCTION OF ACTIVE PHARMACEUTICAL INGREDIENTS
}

\author{
Annam Renita A. ${ }^{1}$, Shyam Saxena ${ }^{2}$, Deepak. K. Shukla ${ }^{3}$ \\ ${ }^{1}$ Research Scholar, Sathyabama University, Jeppiaar Nagar, Rajiv Gandhi Road, Chennai, \\ ${ }^{2}$ Student ,Department of Chemical Engg., Sathyabama University, Jeppiaar Nagar, Rajiv Gandhi Road, Chennai, \\ ${ }^{3}$ Ranbaxy Laboratories Limited \\ E-mail : 'renichris@yahoo.com
}

\begin{abstract}
Properties of drug substances and dosage forms can be highly affected by the particle size, a critical process parameter in pharmaceutical production. The fundamental issue with particle size analysis is the variety of equivalent particle diameters generated by different methods, which is largely ascribable to the particle shape and particle dispersion mechanism involved. Thus, to enable selection of the most appropriate or optimal sizing technique, cross-correlation between different techniques may be required. This review offers an in-depth discussion on particle size analysis pertaining to specific pharmaceutical applications and regulatory aspects, fundamental principles and terminology, instrumentation types, data presentation and interpretation, in-line and process analytical technology. For illustration purposes, special consideration is given to the analysis of FXD.
\end{abstract}

KEYWORDS : Pharmaceuticals, Analytical Technology, FXD.

\section{INTRODUCTION}

The application of power ultrasound to chemical processes is one of a number of intensification technologies that have undergone serious and wide ranging development over the past $10-15$ years. In these years emphasis of chemical companies have been on environmentally clean technology and to minimize energy usage . the use of ultrasound offers possibilities for cleaner reactions through improved product yield and selectivity, and for enhanced product recovery and quality through application to crystallization and other product recover and purification processes

The first application of ultrasound to crystallization (sonocrystallization) was in 1927 . interested in the application of ultrasound to crystallization in pharmaceutical and fine chemicals sectors of industry has gained momentum in recent years with the increased focus on specificity of effect, and the corresponding requirement to prepare and purify complex chemical entities to very exacting standards. Ultrasound may influence crystallization through the mechanism of cavitations and acoustic streaming . sonocrystallization is used to influence crystal size distribution, assist in morphological control, elimination of impurities in the crystal, and improve solid-liquid separation behavior.

On other hand the earliest serious studies of the chemical effect of ultrasound were carried out in the 1950s, where the production of $\mathrm{H}_{2} \mathrm{O}_{2}$ was reported as evidence of radical formation on insonation of aqueous solutions. The more recent interest commenced with the outstanding enhancements that were observed in organometallic reactions and the field has expanded and developed dramatically with an increasing volume of literature publications and conference proceedings. It has helped to increase the productivity and product quality. However, whilst the concept of ultrasonic processing is not new, the ability to use it on an industrial scale is indeed recent advances in equipment have made its implementation at industrial scale.

\section{AIM AND SCOPE OF PRESENT INVESTIGATION}

\section{A. Effects of ultrasound on crystallization}

Effect of ultrasound has been observed in improving the Crystallization of organic compounds of low to medium molecular weight. For example crystallization improvement have been reported for paroxetine, aspartame, adipic acid, amino acids etc. Larger molecules, such as proteins an peptides, and highly water soluble / polar compounds such as sugars are more demanding because of the difficulties in inducing crystal nucleation and the large super saturation driving forces involved. Literature reports indicate that ultrasound has been employed increasingly in the preparation of amorphous and nanostructured materials. These developments are potentially important in showing that ultrasonic technology can be used increasingly in new areas with considerable commercial potential and indeed may lead to a greater understanding of sonocrystallision with respect to the observed effects on product structure and morphology.

\section{B. Nucleation of crystals by ultrasound}

It has been told that ultrasound helps in improving crystallization properties. But now matter under consideration is how it works. It is usual to consider crystallization in terms of the fundamental processes of nucleation and crystal growth whilst such events will occur sequentially in any crystallization process, they can be very difficulty to decouple for fundamental investigations. 
Although nucleation theories have advanced considerably in recent years, the tem plating or a particular ordering within the solid state via the nucleation process is not fully understood. Ultrasound can induce primary nucleation in nominally particle-free solutions and, noteworthy, at much lower super saturation levels than would otherwise be the case. The application of ultrasound to a liquid induces the phenomenon of CAVITATION in the process fluid. Indeed, most sonochemical and sonoluminescent effects of ultrasound have been attributed to transient cavitation. Thiscavitation effect creates bubbles during successive cycles of compression and rarefaction to a point of transient bubbles collapse, perhaps after only two acoustic cycles. This bubble collapse produces highly resolved regions of extreme excitation, temperature (5000 K) and pressure (2000 atm), as well as concomitant release of shock waves. The region why such local and transient energy concentrations correlate with nucleation events have yet not been fully explained. In one sense the effect is counter- intuitive, in that a local temperature increase will reduce or eliminate the

Supersaturation in the immediate vicinity, effectively removing the driving force to nucleation. However the shock waves may contribute to nucleation in the region of supersaturated solution somewhat remote from the cavitation event.

Other postulate suggest:

(1) subsequent rapid local cooling rates, calculated at $107-1010 \mathrm{~K}, \mathrm{~s}-1$, play a significant part in increasing super saturation .

(2) localized pressure increases reduce the crystallization temperature.

(3) The cavitation events allow the excitation energy barriers associated with nucleation to be surmounted.

So in this case it should be possible to correlate the numbers of cavitation and nucleation events in quantitative way.

There is clearly a need for further research on the relationship between cavitation and nucleation. Interestingly, it has been suggested that nucleation caused by scratching the walls of a vessel containing a supersaturated solution with glass rod/spatula could be the result of cavitation.

In many respects, the ease or difficulty of carrying out a crystallization process can be linked to an understanding of the metastable zone (MZ). For a cooling crystallization, the width of this zone (MZW) can be described as the temperature drop below the solubility curve at which the solid starts to separate spontaneously for a given super saturation level and cooling rate(sopersolubility limit).

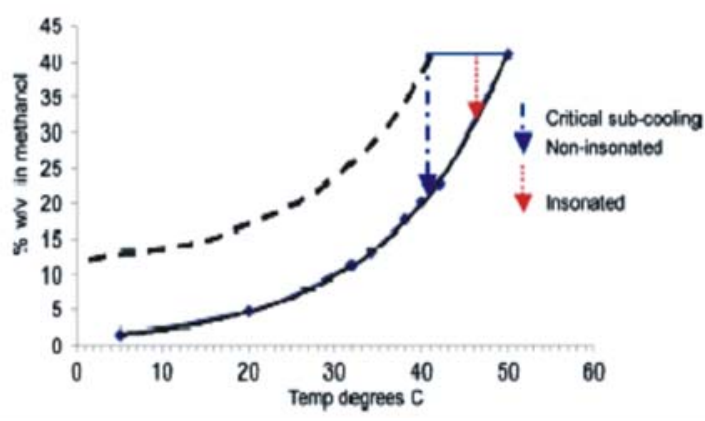

Fig .1 crystallization of sorbitol hexaacetate in methanol.

Sonocrystallization helps in reducing the width of metastable zone. Figure 1 shows the effect of a short $30 \mathrm{~s}$ burst of high-intensity $20 \mathrm{kHz}$ ultrasound on the crystallization of sorbitol hexaacetane from methanol where the MZW has been narrowed by some $5 \mathrm{C}$, as indicated by the observation of crystals at lower levels of super saturation.

One of the important use of ultrasound in crystallization is that it provides great flexibility in choosing crystal size according to the need of industry. It is possible to " tailor " a crystal size distribution between the extreme cases by choosing different mode of ultrasound exposure. Basically it can be divided in three modes of exposure.

1) Giving a short burst of ultrasound to nucleate at lower levels of super saturation i.e using insonation just to initiate the process and then allow to grow them. This will produce biggest size of crystals.

2) Giving continuous (or perhaps a longer single burst) insonation throughout the duration of process produces a many nuclei resulting a small crystals.

3) Pushed or intermittent application of ultrasound can give intermediate effects.

In any of these events, the optimum needs to be determined by experimental investigation. Particle size control has been demonstrated for a number of molecules including sorbitol hexaaceetate, where more regularly shaped crystals can be formed and adapic acid.

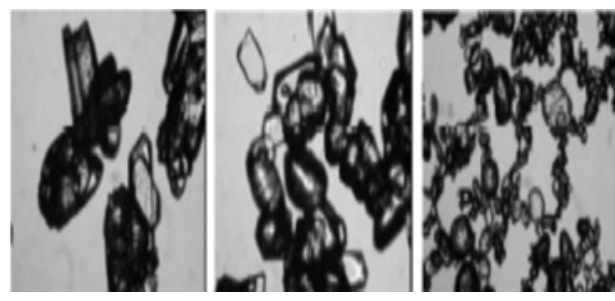

Fig .2 Cooling Crystallization of Adapic acid from water :( left ) 
Fig .2 Illustrates the effect of ultrasound on the overall shape and size of crystals of Adipic acid. Non insonated control experiment : (center) short ultrasound burst at $48 \mathrm{c}$ (right) continous insonation.

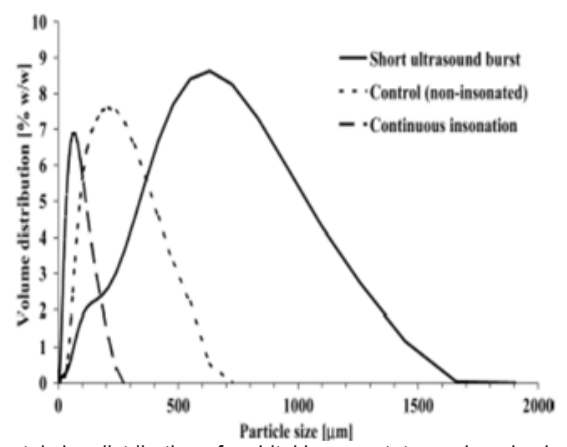

Fig .3 Crystal size distribution of sorbitol hexaacetate produced using different ultrasonic process conditions.

The effect of ultrasound on the particle size distribution for sorbitolhexaacetate is shown in figure 3 . it is possible that ultrasound may also induce secondary nucleation by mechanically disrupting crystals or loosely bound agglomerates that have already formed. A number of new active pharmaceutical ingredients (APIs) have also been examined; one particular small molecule has been shown to exhibit troublesome behaviour in terms of crystal habit. When high (labile) levels of supersaturation were reached in a standard cooling crystallization, high nucleation rates, along with concomitant poorly controlled crystallization, led to the proliferation of a distinct needle habit (Fig 4 ), manifest itself in poorly stirred slurries and variable product bulk density. Conversely, when a solution was treated with ultrasound at much lower level of supersaturation, a high desired rhombic-/plate-type habit was easily produced.

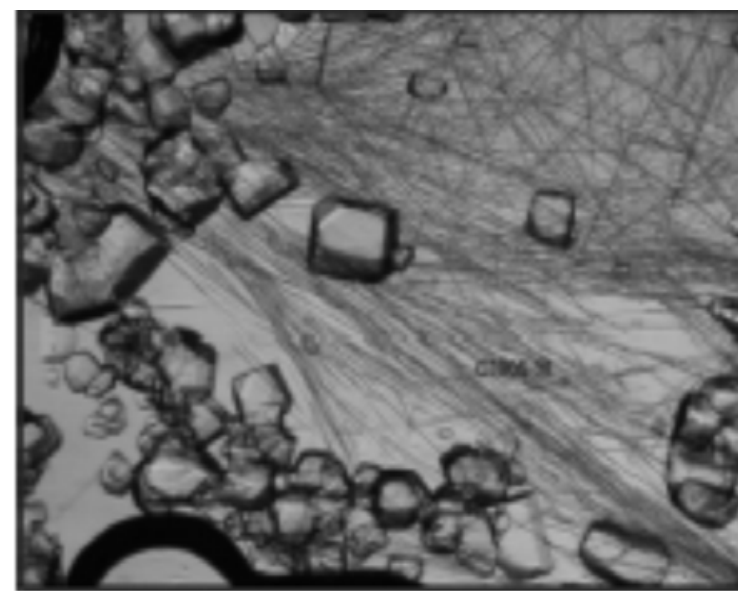

Fig .4 growth of two different crystal habits of a small molecule API at labile level of supersaturation.

\section{Polymorphism and ultrasound}

Polymorphism is common amongst organic materials, and given that small- molecule drugs can be flexible and exhibt significant internal freedom. Indeed, isonation of the " wrong" polymorph brings substantial problems in pharmaceutical applications. Judicious applications of ultrasound to a polymorphic system at the right level of supersaturation can assist in isolating the ground-state polymorph (the most thermodynamically favoured and least soluble ) or one near the ground state. Conversely, without ultrasound (or careful seeding ) one may expect to generate a kinetically favoured and more soluble metastable polymorph. Narrowing of the MZW for the more stable polymorph can lead to nucleation at lower levels of supersaturation than would otherwise be the case and, ideally, undersaturated with respect to the less stable polymorph. Supersaturation can then be managed over the process cycle, such as with slow or exponential cooling, so as to avoid indiscriminate nucleation. In crystallization processes induced by the addition of an antisolvent, where high supersaturation levels may be produced very rapidly, it has been shown that the application of ultrasound reduces not only the induction time of nucleation but also the spread of variability in induction time at a given level of supersaturation. Typically in antisolvent can be used in conjunction with ultrasound to induce crystallization in a controlled manner.

\section{Control options}

In ultrasound crystallization there are number of control options available :

1) Frequency: It changes the size of cavitation event affecting unit cell formation.

2) Amplitude: It affects the ground state selection.

3) Cavitation Threshold: Acoustic streaming can influences nucleation.

\section{Advantages of Ultrasonic Nucleation over Seeding}

The ultrasonic induction of crystal nucleation is clearly advantageous over seeding. Intentional seeding is common in industrial crystallization process, including the crystallization of APIs, but they usually require operator intervention to add the seeds, which may entail additional engineering to control the seed addition whilst maintaining the containment. The effects of intentional seeding include narrowing of the MZW, shortening of induction times, and control of particle size and distribution. In a batch process, seeds have to be added at precisely the correct time during the development of the supersaturation profile. Addition too soon to a solution that is undersaturated will result in the seeds dissolving. Seeding too late will also be 
ineffective because the solute material may already have rapidly (and possibly disastrously) crystallized as a result of high supersaturation levels with ensuring high nucleation rates, giving a product of inferior physical characteristics. Extremely small seed crystals generated by insonation offer all the advantageous of conventional seeding without many of the drawbacks such as handling, actual physical size of the seed, when to add a batch process, and CGMP quality of the seed. The exact point of nucleation ( in terms of supersaturation) can be well controlled, and to a degree the number of nuclei generated as a result of the prevailing supersation level.

\section{Effect of Ultrasound on Crystal Growth}

The effects of ultrasound on crystal growth have enhanced bulk-phase mass transfer. Insonation of a liquid creates mechanical disturbances via both cavitation and acoustic streaming. Such effects will alter the fluid dynamics and increase bulk-phase mass transfer of solute to the surface to the growing crystal. In most cases, however, the surface nucleation and integration effects at the crystal faces will determine the growth rate of each individual face and, hence, the habit of the crystal. A theoretical study along these lines has suggested that the effects of ultrasound on crystal growth will depend on the magnitude of the superstauration driving force. At low supersaturation, with growth velocities at the crystal faces around 10-10 m,s-1, the application of ultrasound doubled the growth rate, while at higher supersaturation with velocity around 10-7 m, s-1 there appeared to be no effect. The classical Burton-Cabrera-Frank (BCF) theory of crystal growth without insonation postulates that growth rate is limiting by the formation of new surface layers at defect sites and predicts that the growth rate will exhibit approximately quadratic dependence on supersaturation at low supersaturation levels, while at higher levels the dependence becomes closer to linear. The ultrasound effect is explained by the hypothesis that, at low supersaturation, the quantity of available growth units in the vicinity of the crystal surface is small. Under these conditions, bulk-phase mass transfer becomes rate limiting in supplying growth units to the crystal surface, and its ultrasonic enhancement will enhance the growth rate.

\section{Scale-Up and Equipment Development}

The biggest barrier to the adoption of power ultrasound technology in manufacturing has been the lack of suitable equipment for use in industrial environments at the scale required. Most discoveries in sonochemistry and sonoprocessing have been carried out in laboratories on the milligram gram scale using either high-intensity probe or bath systems. There is a fundamental requirement for equipment that may be operated simply and reliably at the kilogram-tone scale in the small-volume manufacturing of fine chemical and pharmaceuticals and importantly in an explosion-proof environment. For bulk -commodity chemicals manufacturing, scale of at least an order of magnitude larger than this would be required. Although the cost-benefit basis of the technology makes it less attractive for this type of application, all processes should be examined on a case by case basis. A simple system incorporates a crystallizing jacketed vessel and a processes flow loop with glass flow cell containing an ultrasonic probe. That said, laboratory probe and bath systems are usually built with a single ultrasonic transducer attached either to a delivery tip (probe) or to the base of a cylindrical or rectilinear vessel (bath). The size of these devices limits the scale at which they may be operated. In addition they also suffer further disadvantages with respect to the distribution of the energy intensity that they deliver. A bath will deliver nonhomogeneous acoustic fields throughout the medium with maximum amplitude at multiples of the half wave length of sound. The nonhomogeneity of the acoustics field means that one must be careful with the positioning of the reaction/crystallizing vessel (depth, positioning with respect to where the transducer are mounted) if direct comparison is to made for a series of experiments. In addition, the higher power level will be at points closest to the base and will dissipate with increasing distance from the transducer.

Most desired effects of ultrasound are mediated by cavitation, which occurs at a threshold energy density. Above this threshold, the number density of cavitational events increases, but the increase is less proportional to the increase in energy input, and the effect levels off at very high input levels. The cavitatonal threshold varies with the conditions of operation, including the physical properties of the liquid being insonated. Energy input density of around $35 \mathrm{~W}$, is a reasonable rule-of-thumb to exceed the cavitational threshold for most common solvents operating at room temperature. Probe systems deliver very high intensity at the tip of the probe, the point of delivery, but the energy density is concentrated in the axial direction, away from the tip, and falls away rapidly with distance according to an inverse square law. Indeed, it is not possible to transmit an intense cavitation field more than $2-5 \mathrm{~cm}$ beyond the end of the probe, nor is it a suitable means to transmit the acoustic energy into large process volume, thus precluding scale-up. Probe systems only work effectively if operated in a geometry where most of the working liquid is constrained within the longitudinal high-intensity region or where the liquid is stirred vigoursly. In addition they suffer from erosion and particle shedding at the delivery tip surface, they may also be subject to 
cavitational blocking(acoustic decoupling), and the large transducer displacement increases stress on the material of the construction, resulting in the possibility of failure. Whilst good for laboratory studies, they cannot be used at large commercial scale. Early the scale-up designs extended to two or three transducer but more recent developments in both transducer design and bonding technology have allowed the number of transducer fitted to an installation to be increased well beyond this. Another aspect of scale-up is that it effects specific. Different applications depend on different effect arising from ultrasound, and systematic scaling requires that the effect that is important for the particular application be identified, measured, and subsequent used as the basis for scaling. Crystallization effect will be determined principally by the enhancement of the nucleation rates, which as a first approximation may be assumed roughly proportional to the number density of cavitation events. Thus, the measurement of acoustic intensity is a major point of consideration, and indeed several methods are available. These include the calorimetric method (whilst simple, this can be rather inaccurate), radiometric method (measurement of a force exerted on a surface by a radiation pressure / parallel sound beam ), and measurement of the acoustic pressure using calibration hydrophones. Acoustic pressure measurement can be very useful due the ease of the hydrophones and the fact that fast Fourier transform analysis can be performed which provides information on the type of cavitation taking place.

\section{LARGE - SCALE PROCESSING EQUIPMENT}

Technology for the scale - up of ultrasound processing have developed in many different ways, which may be classified generally as

1) probes, i.e., tips or other small areas devices delivering very high local intensities, in a flow cell or large volume

2) opposing parallel transducers arranged around a duct, through which the process solution or suspention flows.

Probe system have been developed that employ a high intensity probe operating in a flow cell, or immersed in a large volume. The probe is in direct contact with the process fluid, but in such systems the acoustics energy is not particularly well focused other than at the tip. systems where opposing parallel plate transducers are used to insonate a fluid flowing through a duct contain the acoustic energy to a greater extent than a probe system; however, the energy is still not particularly well focused to a central volume. Devices where an annular duct is employed through which the process fluid flows and around which transuducers are placed may be considered to be similar to an opposing parallel plate arrangement where limiting focusing of ultrasound occurs. In terms of energy efficiency, the optimum design of equipment would deliver ultrasonic energy intensity slightly above the cavitational threshold throughout its working volume. A number of developments have concentrated on utilizing a central resonating duct through which the process fluid flows; one such system uses only one transducer, whilst others, such as the sodeva sonitube, use several. The essential features of this device is that the dimensions of the tube are designed to give standing waves along its length. Resonating systems essentially set up standing waves using coherent ultrasound within the working liquid and will therefore give rise to spatially inhomogeneous and very often high power densities. Although a resonating tube will result in acoustic energy being transmitted into the fluid from the entire inner surface of the tube, the focusing effect is limited as is the achievable power.

The alternative approach to the design of scale-up units for industrial application is to avoid resonance and standing waves and to develop systems that avoid coherent wave relationships. This equipment designed and manufactured by C3 Technology for sonoprocessing has following this course. There are a number of advantages in using noncoherent ultrasound; the more even distribution of the ultrasound through the working fluid is a key benefits. In addition one can design equipment with greater flexibility in terms of dimensions, frequency, and configuration. We embarked on noncoherent designs after our early experience with multitransducer system gave rise to difficulties with transducers tuning in to each other and of mechanical resonance in system components. Such difficulties can undoubtedly be more easily overcome with modern transducers and system designs. The original multitransducer designs were based on a 4-5-L insonated volume as a cylindrical duct, $120 \mathrm{~mm}$ in diameter, fitter with three radially mounted transducers. To reduce surface erosion and mechanical stresses at the point of contact, a liquid barrier was employed between the transducer and the (thinned) duct wall. This unit was design for noncoherent ultrasonic operation with a nominal frequency of $20 \mathrm{KHZ}$. Three transducers, tuned to slightely different frequencies between 19 and $21 \mathrm{KHZ}$, indicated that the power input densities were reasonably uniform throughout the $4-5 \mathrm{~L}$ working volume.

Most recent developments have employed direct bonding of the transducer to the surface of the vessel. Improvements in the bonding method, and a move to a transducers with low individual outputs, have enabled the move to systems with large numbers of transducers to give 
an acoustic pattern that is uniform and noncoherent above the cavitational threshold throughout the working volume. The use of low output transducers gives the additional advantage of avoiding the phenomenon of cavitational blocking (acoustic decoupling), which arises where power densities close to the delivery points are very high. In addition these munltitransducer units very effectively concentrate ultrasonic intensity towards the central axis of the cylinder and away from the vessel walls, thus reducing problems of erosion and particle shedding. This vessel can be operated in batch mode or, for larger - scale work, in continuous mode whereby units can be combined in a modular fashion for "scale-out" and increased residence time. In summary, a plurality of low electrical and acoustic power (-1-3, cm ) transducer produces $25-150 \mathrm{~W}, \mathrm{~L}-1$, but ideally 40-80 W,L-1. the power can be applied continuously or pulsed. A typical example of the flow cell is shown in fig .5

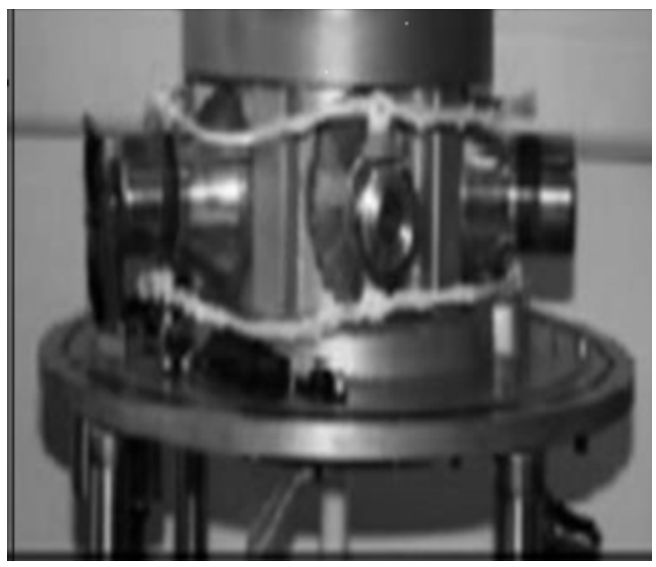

Fig .5 Ultrasonic Flow Cell.

The Objective is to study the effect of ultrasound wave on API-F sample by sono- crystallization.

\section{Following Paramenters:}

1) Temperature

2) Flow rate

3) Time

\section{Varying parameters:}

4) Concentration

a) Regular sample

b) 5-times diluted sample

5) Amplitude
a) 25
b) 50
c) 75
d) 100

6) Nucleation

\section{PROCEDURE}

Crystalline API-F sample was charged in the reactor vessel ( 2 l ) being pumped to the sonocrystallizer which converts the electrical energy into ultrasound energy. ultrasound waves thus produced is allowed to act on the slurry of API-F sample. Due to the impact of ultrasound waves on API-F, the particle size gets reduced. The samples are then taken after an interval of one hour. This process is repeated for different amplitude readings ranging from $25 \%$ to $100 \%$.

\section{RESULTS AND DISCUSSION}

\section{API normal sample photographs without dillution}

\section{Photographs of the API sample not subjected to ultrasound waves}

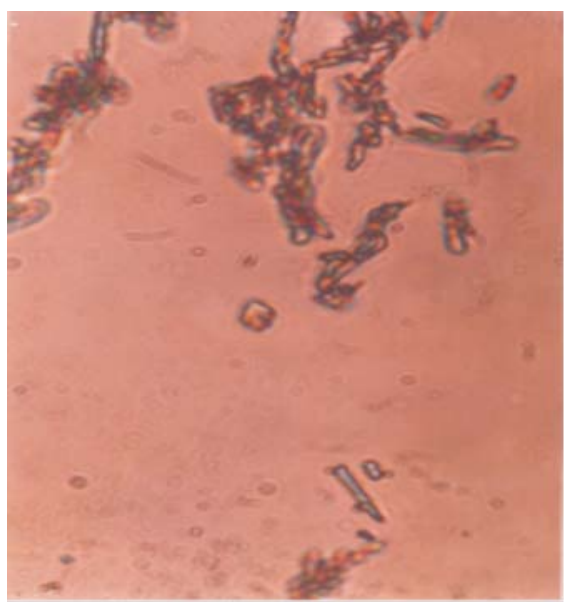

\section{Photographs of the API sample subjected to ultrasound waves at $25 \%$ amplitude}

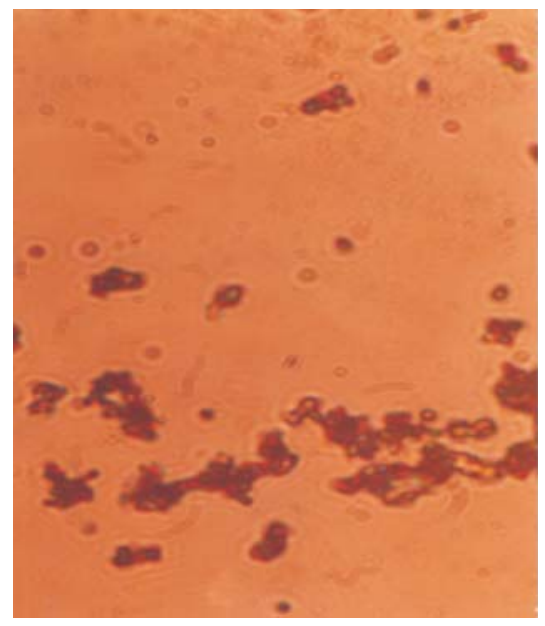

Photograph of the API sample which was taken after $1 \mathrm{hrs}$ of ultrasound wave impact 
Photographs of the API sample subjected to ultrasound waves at $25 \%$ amplitude

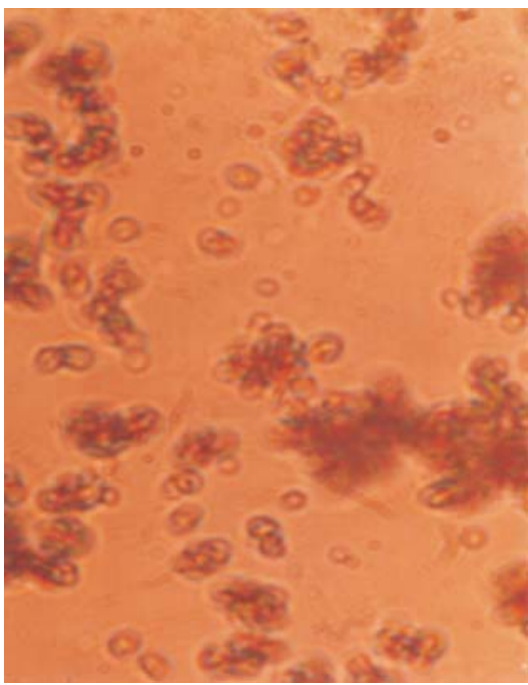

Photograph of the API sample which was taken after $2 \mathrm{hrs}$ of ultrasound wave impact

Photographs of the API sample subjected to ultrasound waves at $25 \%$ amplitude with decreased concentration ( diluted up to 5 times)

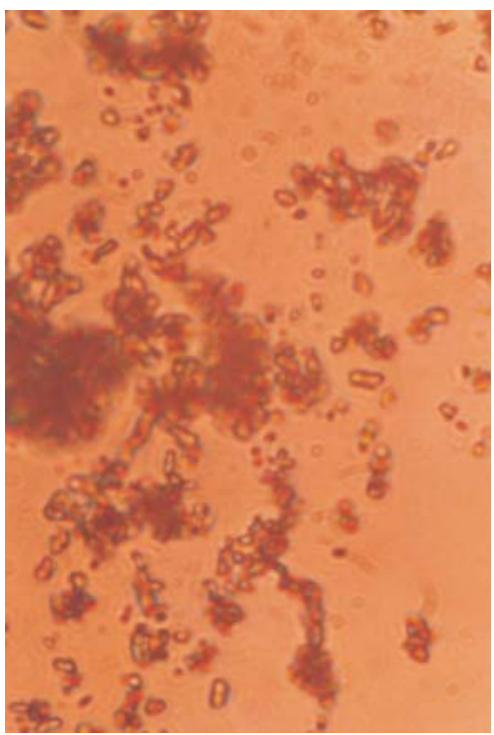

Photograph of the API sample which was taken after $1 \mathrm{hrs}$ of ultrasound wave impact
Photographs of the API sample subjected to ultrasound waves at $25 \%$ amplitude with decreased concentration (diluted up to 5 times)

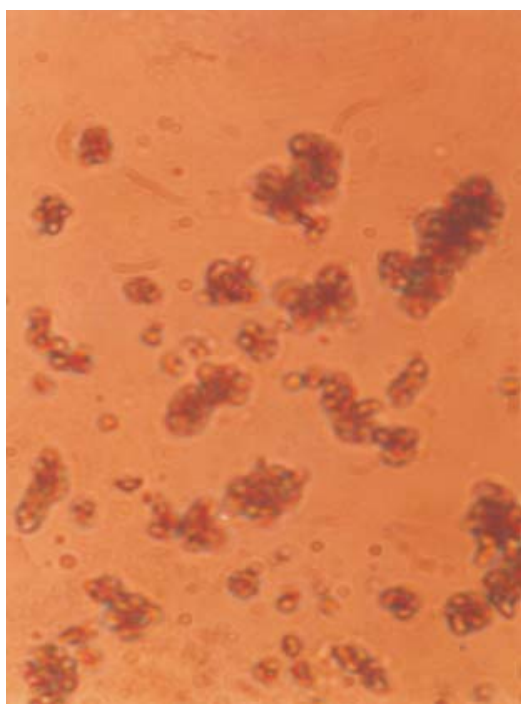

Photograph of the API sample which was taken after 2 hrs of ultrasound wave impact

\section{Photographs of the API sample subjected to ultrasound waves at $50 \%$ amplitude}

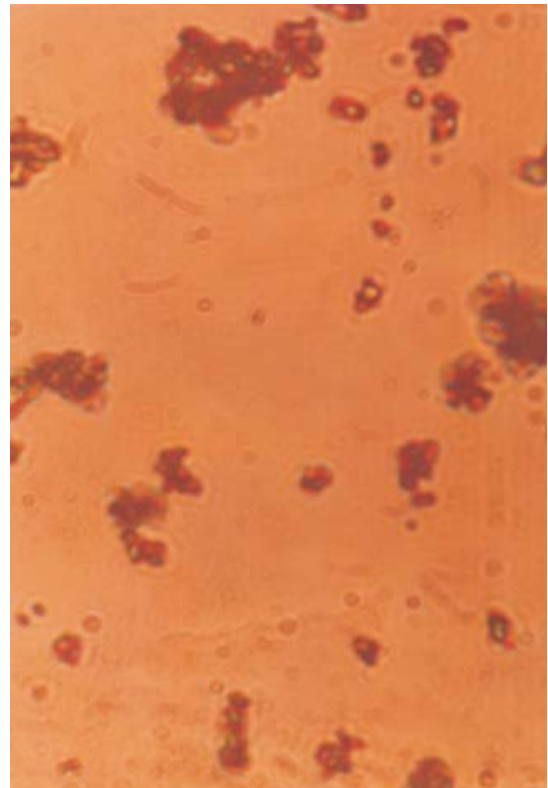

Photograph of the API sample which was taken after 2 hrs of ultrasound wave impact 
Photographs of the API sample subjected to ultrasound waves at $50 \%$ amplitude

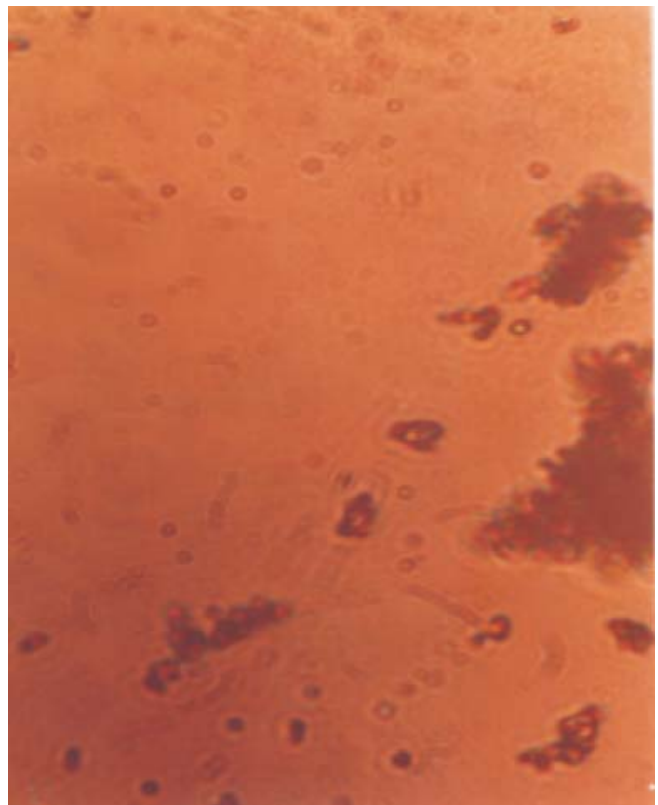

Photograph of the API sample which was taken after 3 hrs of ultrasound wave impact

Photographs of the API sample subjected to ultrasound waves at $50 \%$ amplitude with decreased concentration ( diluted up to 5 times)

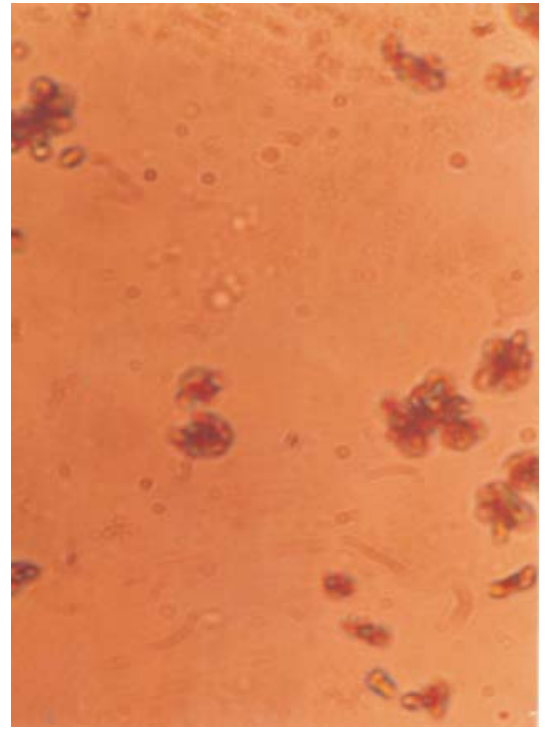

Photograph of the API sample which was taken after 2 hrs of ultrasound wave impact
Photographs of the API sample subjected to ultrasound waves at $50 \%$ amplitude with decreased concentration (diluted up to 5 times)

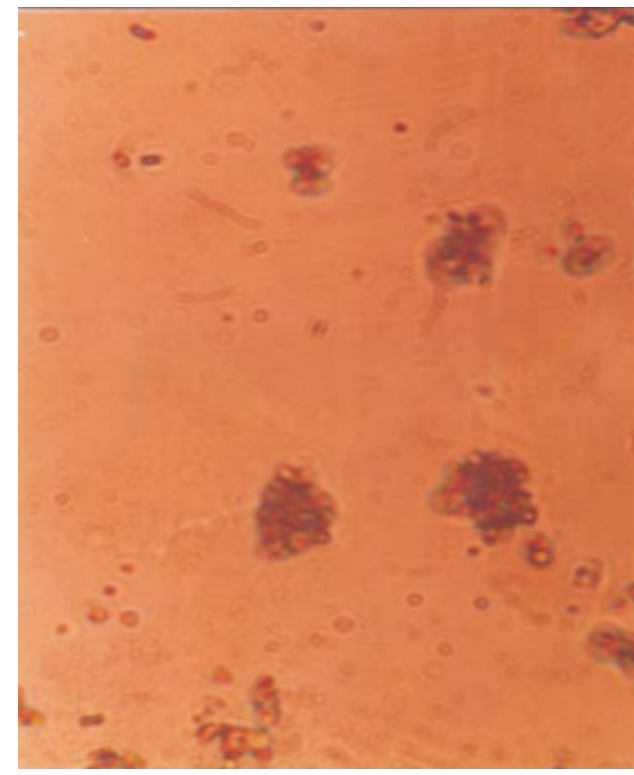

Photograph of the API sample which was taken after 3 hrs of ultrasound wave impact

Photographs of the API sample subjected to ultrasound waves at $75 \%$ amplitude

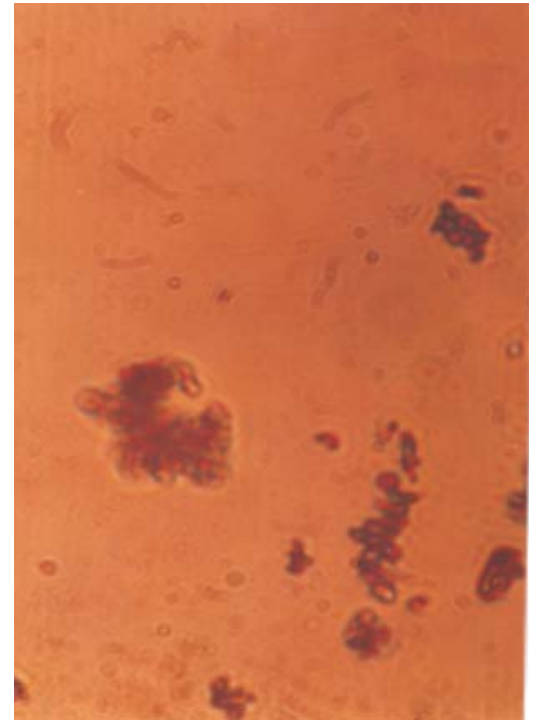

Photograph of the API sample which was taken after 2 hrs of ultrasound wave impact 
Photographs of the API sample subjected to ultrasound waves at $75 \%$ amplitude

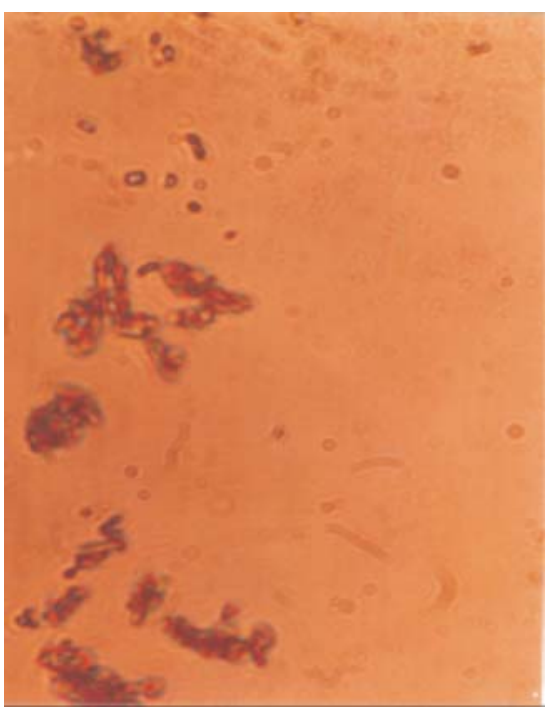

Photograph of the API sample which was taken after 3 hrs of ultrasound wave impact

Photographs of the API sample subjected to ultrasound waves at $75 \%$ amplitude with decreased concentration ( diluted up to 5 times)

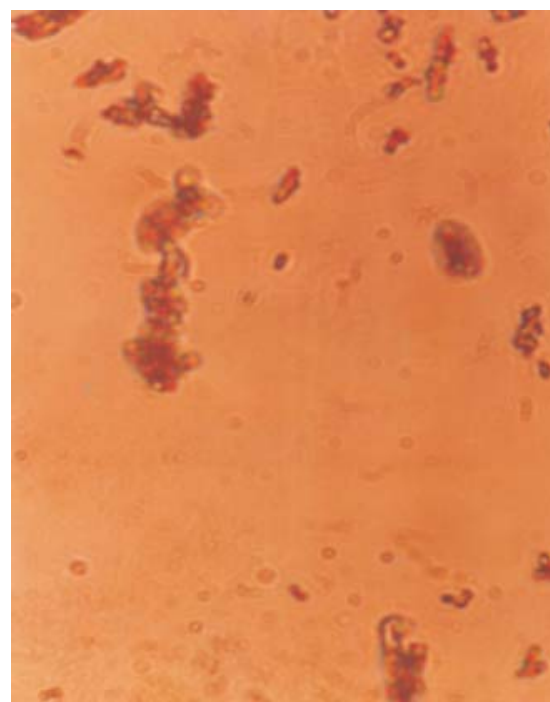

Photograph of the API sample which was taken after 2 hrs of ultrasound wave impact
Photographs of the API sample subjected to ultrasound waves at $75 \%$ amplitude with decreased concentration (diluted up to 5 times)

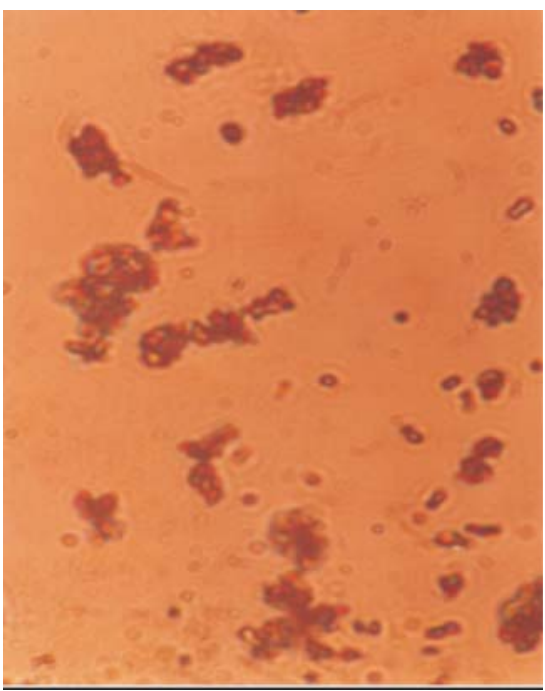

Photograph of the API sample which was taken after $3 \mathrm{hrs}$ of ultrasound wave impact

Photographs of the API sample subjected to ultrasound waves at $100 \%$ amplitude

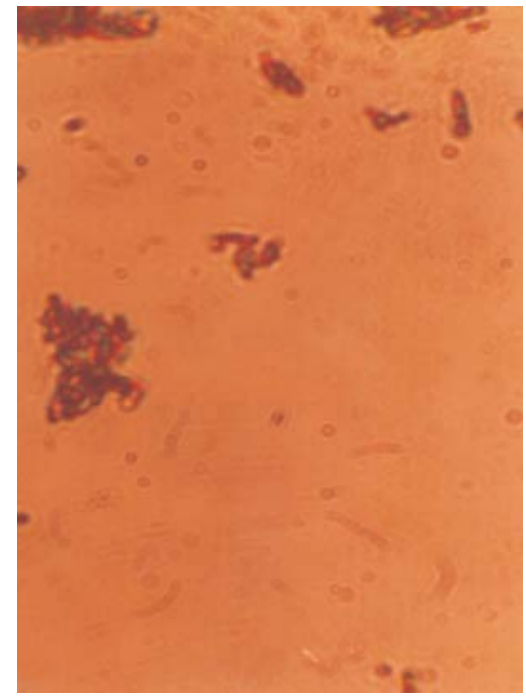

Photograph of the API sample which was taken after 2 hrs of ultrasound wave impact 


\section{Photographs of the API sample subjected to ultrasound waves at $100 \%$ amplitude}

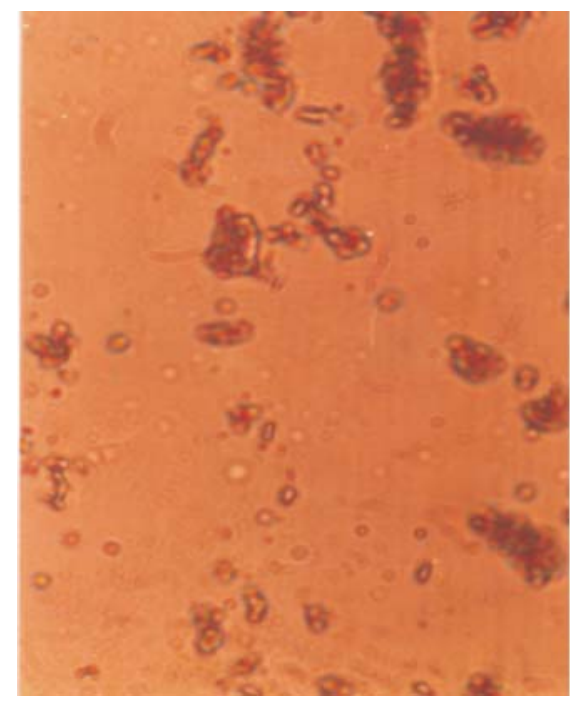

Photograph of the API sample which was taken after 3 hrs of ultrasound wave impact

Photographs of the API sample subjected to ultrasound waves at $100 \%$ amplitude with decreased concentration (diluted up to 5 times)

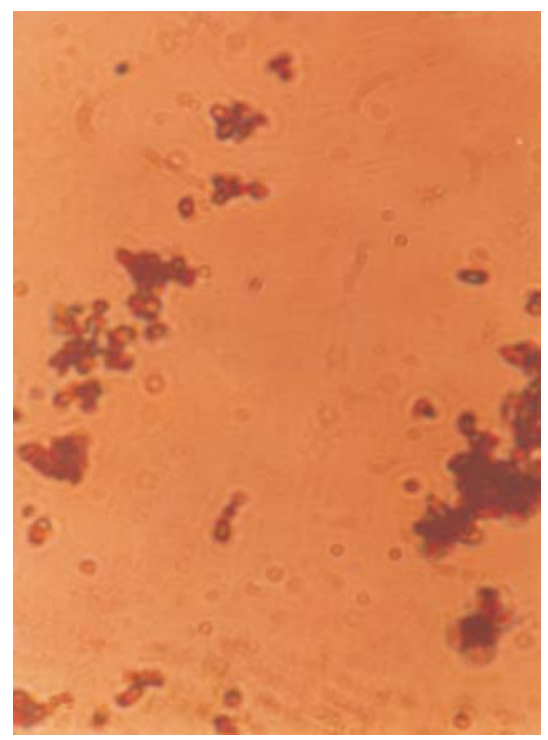

Photograph of the API sample which was taken after $2 \mathrm{hrs}$ of ultrasound wave impact
Photographs of the API sample subjected to ultrasound waves at $100 \%$ amplitude with decreased concentration (diluted up to 5 times)

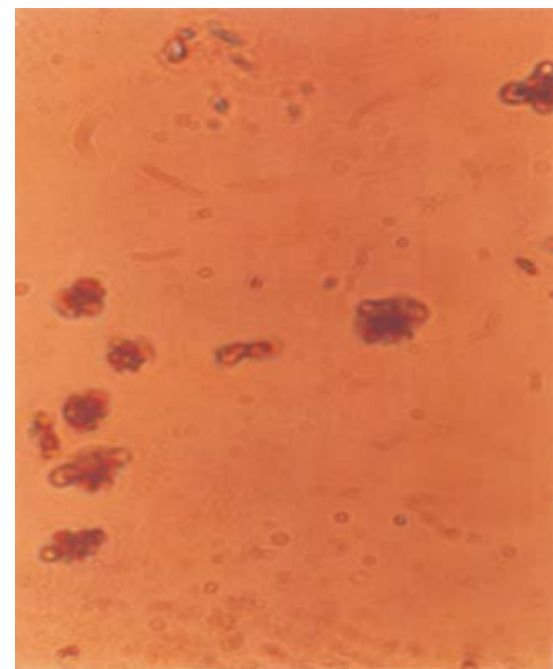

Photograph of the API sample which was taken after 3 hrs of ultrasound wave impact

\section{Other Applications of Ultrasound}

Besides use of ultrasound in sonocrystallistion and sonochemistry it may also be used in many other processes which in turn provides efficient and environment friendly solutions.

\section{Mixing procedures}

A very important application of ultrasound is in mixing and homogenizing, emulsifying, suspending, and dispersing of various products. Ultrasound can be used to produce emulsions, e.g. petroleum spirit-water mixtures. Moreover, depending on the specifics of the application, ultrasound can be used to degas a medium or enrich it in oxygen or ozone. It is even possible to deform liquids in specific applications.

Mixing ratios in the microbe realm become possible with ultrasound cavitaion flags at the ultrasonic oscillator not only in the laboratory, also in industrial applications the ultrasonic systems are used to emulsify, disperse, homogenize and suspend. Small disintegration system DG 100, which is used particularly in laboratories.

\section{Biotechnology}

In biotechnology, ultrasound is applied both for specific activation (or deactivation) of enzymes and for manipulation and separation of biological cells by purposeful disintegration of their content. As an example, ultrasound can be used to accelerate the extraction of flavors and other substances from plant-derived products.

In certain applications, ultrasound has proven itself as an environment friendly substitute for chlorinated solvents and disinfections agents. Disinfection can be achieved by dispersing accumulated bacteriaand reducing the level ofbactericide. 


\section{Disintegration of particles}

High performance ultrasound can be used to reduce the particle size of APIs, minerals, powders, paints, and lacquers. The application of ultrasound for disintegration of paint pigments in the printing and toner industry or for pharmaceutical products is utilized on an industrial scale in many places. Even chocolate particles for liqueurs have been reduced in size with this technology, which demonstrates even to the food-processing industry.

\section{Water treatment}

Another application from the realm of environmental engineering is the use of ultrasound in potable water treatment. Ultrasound is used in this application to support common procedures such as UV irradiation and ozone treatment.

Ultrasound is also often used in places in which it is essential to keep bodies of water free from algae or other biological cultures. Ultrasound provides an extremely environment-friendly procedure since it requires no chemical activities. Beside all these applications ultrasound can be used in wire cleaning facilities, ultrasound-based cutting facilities, atomizing/fog generating facilities, and many more.

\section{CONCLUSION}

The API sample which was subjected to ultrasound waves had a reduction in its size as the percentage amplitude was increased. The purpose of this experiment was to generate reduced API- particle size to widen the scope of usage of the API sample. Thus this technology can be industrially applied to meet the consumers requirement.

\section{REFERENCES}

[1] N. Blagden, M. de Matas, P.T. Gavan, P. York, Crystal engineering of active pharmaceuticalingredients to improve solubility and dissolution rates, Advanced Drug Delivery Reviews (2007) 10 May.

[2] Aulton M.E., Pharmaceutics, The science of dosage form design, 2nd edition, Churchill Livingstone, London, 2002, 113-138, 234-252.

[3] Michael Hite, Lead Research Associate, Stephen Turner, Oral Delivery of Poorly Soluble Drugs400, Pharmaceutical Manufacturing and Packing Sourcer Summer '03 issue. Samedan Ltd. 2003.

[4] Sheere Banga, Garima Chawla and Arvind K Bansal, New Trends in the Crystallisationof ActivePharmaceuticallngredients,Businessbriefin $\mathrm{g}:$ Pharmagenerics, 2004, 70-74.

[5] Irene Pasquali, Ruggero Bettini, Ferdinando Giordano, Solid-state chemistry and particle engineering with supercriticalfluids in pharmaceutics, European journal of pharmaceutical sciences 2006, 27, 299-310.

[6] A.M. Juppo, C. Boissier, C. Khoo, Evaluation of solid dispersion particles prepared by SEDS, Int. J. Pharm., 2003, 250, 385-401.

[7] Behrend, O., Schubert, H.: Influence of hydrostatic pressure and gas content on continuous ultrasound emulsification, in: Ultrasonics Sonochemistry 8 (2001) 271-276.

[8] Formation of Nanoparticles of a Hydrophilic Drug Using Supercritical Carbon Dioxide and Microencapsulation for Sustained Release . Disease-a-Month , Volume 51 , Issue 6 , Pages 362 -373A. Thote, R. Gupta

[9] A.M. Juppo, C. Boissier, C. Khoo, Evaluation of solid dispersion particles prepared by SEDS, Int. J. Pharm., 2003, 250, 385-401.

[10] W.Y. Lo, S.L. Law, Dissolution behavior of griseofulvin solid dispersions using polyethylene glycol, talc, and their combination as dispersion carriers, Drug Dev. Ind. Pharm., 1996, 22, 231236.

[11] D.Singhal,W. Curatolo, Drug polymorphism anddosage form design: a practical perspective,Adv. Drug. Deliv. Rev., 2004, 56, 335347.

[12] Vasu Kumar Kakumanu and Arvind K Bansal, Supercritical Fluid Technology in Pharmaceutical Research, Businessbriefing: Labtech, 2004, 71-73.

[13] RiteshSanghvi, Daniel Evans, Samuel H. Yalkowsky, Stacking complexation by nicotinamide:A useful way of enhancing drug solubility, International Journal of Pharmaceutics, 2007,336,

[14] St'ephaneGibaud, Siham Ben Zirar, Pierre Mutzenhardt, Melarsoprol-cyclodextrins inclusion complexes, International Journal of Pharmaceutics, 2005, 306, 107-121.

[15] Joseph T. Rubino,Cosolvents and Cosolvency, 2002, 1, 658-670

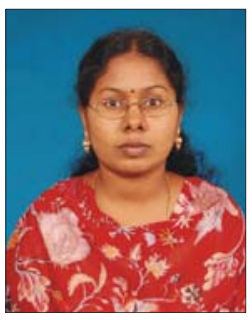

Ms. Annam Renita is a Faculty in the Department of Chemical Engineering, Sathyabama University. Her area of Research work is in Air Pollution Control, activated carbon and Fluidisation Engineering. She is working in this area for the past 4 years. 